\title{
Aversive Stimuli Alter Ventral Tegmental Area Dopamine Neuron Activity via a Common Action in the Ventral Hippocampus
}

\author{
Ornella Valenti, Daniel J. Lodge, and Anthony A. Grace \\ Departments of Neuroscience, Psychiatry, and Psychology, University of Pittsburgh, Pittsburgh, Pennsylvania 15260
}

Stress is a physiological, adaptive response to changes in the environment, but can also lead to pathological alterations, such as relapse in psychiatric disorders and drug abuse. Evidence demonstrates that the dopamine (DA) system plays a role in stress; however, the nature of the effects of sustained stressors on DA neuron physiology has not been adequately addressed. By using a combined electrophysiological, immunohistochemical and behavioral approach, we examined the response of ventral tegmental area DA neurons in rats to acute as well as repeated stressful events using noxious (footshock) and psychological (restraint) stress. We found that aversive stimuli induced a pronounced activation of the DA system both electrophysiologically (population activity; i.e., number of DA neurons firing spontaneously) and behaviorally (response to psychostimulants). Moreover, infusion of TTX into the ventral hippocampus (vHPC) reversed both behavioral and electrophysiological effects of stress, indicating that the hyperdopaminergic condition associated with stress is driven by hyperactivity within the vHPC. Therefore, the stress-induced activation of the DA system may underlie the propensity of stress to exacerbate psychotic disorders or predispose an individual to drug-seeking behavior. Furthermore, the vHPC represents a critical link between context-dependent DA sensitization, stress-induced potentiation of amphetamine responsivity, and the increase in DA associated with stressors.

\section{Introduction}

The dopamine (DA) system is historically associated with reward-related events (Schultz et al., 1997) and drug abuse (Henry et al., 1989; Pierce and Kalivas, 1997; Lodge and Grace, 2008). Nonetheless, neurochemical studies demonstrated that this system is also activated by maintained stressful stimuli (Imperato et al., 1993; Piazza and Le Moal, 1998). However, to date electrophysiological studies have not shown a response to stress that is consistent with these neurochemical measures; thus, upon exposure to a brief noxious stimulus, DA neurons exhibit a transient inhibition in firing (Schultz and Romo, 1987; Grace, 2000; Ungless et al., 2004) and, in a minority of cases, excitation (Brischoux et al., 2009). In contrast, microdialysis studies indicate that stress elicits a pronounced increase in extracellular DA levels in postsynaptic sites (Thierry et al., 1976; Abercrombie et al., 1989; Kalivas and Duffy, 1995; Saulskaya and Marsden, 1995; Pascucci et al., 2007). Therefore, electrophysiological and microdialysis data appear to be contradictory with respect to aversive

\footnotetext{
Received Oct. 9, 2010; revised Dec. 22, 2010; accepted Jan. 28, 2011.

This work was supported by United States Public Health Service Grant DA15408 to A.A.G. We thank N. MacMurdo and E. Mahar for technical assistance and Brian Lowry for the development of the custom-designed computer software Neuroscope. Brian Lowry was affiliated with the Department of Neuroscience, University of Pittsburgh, Pittsburgh, PA, and employed by Dr. Grace at the time this work was completed.

Correspondence should be addressed to Dr. Ornella Valenti, at her present address: Department of Cognitive Neurobiology, Center for Brain Research, Medical University Vienna, Spitalgasse 4, A-1090 Vienna, Austria. E-mail: ornella.valenti@meduniwien.ac.at.

Dr. D. J. Lodge's present address: Department of Pharmacology; University of Texas Health Science Center, San Antonio, TX 78229.

DOI:10.1523/JNEUROSCI.5310-10.2011

Copyright $\odot 2011$ the authors $\quad 0270-6474 / 11 / 314280-10 \$ 15.00 / 0$
}

event-related DA system responses, and the reason for this discrepancy is unknown. One possible explanation may relate to the time course studied; recordings generally examine the response of single neurons to brief noxious events, whereas neurochemical studies observe changes to maintained stressors measured over a much longer time course. We previously found that maintained alterations within limbic circuits can alter the population activity of DA neurons (i.e., the number of neurons firing spontaneously), an index that was found to correspond to extracellular DA levels measured in the nucleus accumbens (NAc) by microdialysis (Floresco et al., 2003).

One region that controls DA neuron population activity is the ventral subiculum (vSub) of the hippocampus. Thus, activation of the vSub increases DA neuron population activity (Floresco et al., 2001). Moreover, manipulations that increase the behavioral response to amphetamine, such as developmental alterations (Lodge and Grace, 2007) or amphetamine sensitization (Lodge and Grace, 2008), also increase DA neuron population activity via activation of the vSub. The ventral hippocampus (vHPC), and the vSub in particular, is a brain region that is involved in context-dependent processes (Jarrard, 1995; Maren, 1999; Sharp, 1999), and also is considered to be a central mediator of stress responses (Herman et al., 1995). Given that stressors also tend to be context-dependent phenomena, we examined the effects of two types of stressors known to cause activation of the DA system (i.e., repeated footshock and restraint stress) on DA neuron population activity, and whether the vHPC is involved in this response. We show that noxious and psychological stress increase DA neuron population activity, and suggest that this increase can 
account for the increased release of DA in postsynaptic sites to stressors and also to drugs of abuse. Thus, our findings provide an explanation that reconciles divergent data regarding the effect of stress on the DA system. In addition, our results indicate that activation of the DA system could be driven by hyperactivity within the vHPC, as local infusion of TTX into this region reinstated the response to control levels.

\section{Materials and Methods}

Subjects and materials. A total of 181 male Sprague Dawley rats (280-400 g; Hilltop Lab Animals) were used in this study; 129 rats were used for electrophysiological recordings, 10 rats for immunohistochemistry, and 52 rats for behavioral testing. At arrival, rats were housed for at least $5 \mathrm{~d}$ in pairs in a temperature $\left(22^{\circ} \mathrm{C}\right)$ - and humidity $(47 \%)$-controlled facility, with lights maintained on a $12 \mathrm{~h}$ light/dark cycle (lights on at 7:00 A.M.) and food and water available ad libitum.

Pentobarbital sodium (USP) was obtained from Ovation Pharmaceuticals; all other chemicals and reagents were obtained from SigmaAldrich, unless otherwise specified.

Handling of all animals, surgery, and experiment protocols were in accordance with the guidelines outlined in the United States Public Health Service Guide for the Care and Use of Laboratory Animals, and were approved by the Institutional Animal Care and Use Committee of the University of Pittsburgh.

Stress paradigms. The effects of acute, repeated, and/or combined stressors on the activity of ventral tegmental area (VTA) DA neurons were assessed using two distinct stressor paradigms and single-unit extracellular recordings.

In the first study, the effects of a repeated noxious stimulus were examined according to a protocol that has been shown to increase DA levels in postsynaptic sites (Saulskaya and Marsden, 1995) in behaving rats. Thus, 10 repeated electrical stimulations of the sciatic nerve (footshocks) were delivered to the footpad contralateral to the recording site $(3 \mathrm{~mA}$, $1 \mathrm{~s}$; every $1 \mathrm{~min}$ for 10 consecutive minutes) in anesthetized rats.

In a separate group of rats another stimulus that represents a "psychological" stressor was used on awake rats. Rats were restrained in Plexiglas cylinders (custom made; internal diameter $=6 \mathrm{~cm}$, length adjusted accordingly to rat size) for either a $2 \mathrm{~h}$ acute restraint (AR) session or a repeated restraint (ReptR) session, $1 \mathrm{~h}$ restraint each day for 10 consecutive days. In a subset of the last group, the effects of repeated restraint (1 $\mathrm{h} / \mathrm{d}$ for $9 \mathrm{~d}$ ) followed by a subsequent $2 \mathrm{~h}$ acute restraint on the 10th day (combined restraint, CombR) was also evaluated. Single-unit electrophysiological recordings (see below) were performed in anesthetized rats immediately after the stressor for rats that received either AR or CombR, whereas rats receiving ReptR were tested $24 \mathrm{~h}$ after the last stress session.

Surgery and electrophysiological recordings from anesthetized rats. Single-unit extracellular recordings were performed as previously described, with few modifications (Valenti and Grace, 2010). Rats were anesthetized with $8 \%$ chloral hydrate $(400 \mathrm{mg} / \mathrm{kg}$, i.p.) and placed in a stereotaxic apparatus (David Kopf Instruments); core body temperature of $37^{\circ} \mathrm{C}$ was maintained by a temperature-controlled heating pad. Supplemental injections of anesthetic were administered as needed to suppress the hindlimb compression withdrawal reflex. A burr hole was drilled in the skull overlying the recording region in the VTA [in mm from bregma: anteroposterior (AP), -5.3; mediolateral (ML), -0.6], and the dura was resected. Glass electrodes, constructed from $2 \mathrm{~mm}$ outer diameter Omegadot filament glass (World Precision Instruments) and with in situ impedance of 8-12 $\mathrm{M} \Omega$, were lowered slowly into the VTA [dorsoventral (DV), -6 to $-9 \mathrm{~mm}$ ] using a hydraulic microdrive (David Kopf Instruments, model 640). Signals from the recording electrode were amplified by a headstage before being fed into a window discriminator/amplifier ( $1000 \times$ gain, $100-4000 \mathrm{~Hz}$ bandpass; Fintronics Inc.). Signals were also fed into an audio monitor (Grass Instruments, model AM8) and displayed on an oscilloscope (B\&K Precision, model 2120) for real-time monitoring. Data were collected using a data acquisition board interface (Microstar) and monitored on-line using custom-designed computer software (Neuroscope; Brian Lowry, Pittsburgh, PA).
DA neurons were identified with open filter setting (low pass, $50 \mathrm{~Hz}$; high pass, $16 \mathrm{kHz}$ ) and distinguished from other VTA neurons based on their unique long-duration waveform, slow irregular firing rate, and other well established criteria (Grace and Bunney, 1983; Grace et al., 2007). After isolating a DA neuron, 3 min of baseline activity was recorded to determine the baseline firing rate and percentage burst firing. Burst firing was quantified by examining the proportion of action potentials occurring in bursts, or percentage of burst firing, with burst firing defined as the occurrence of two spikes with an interspike interval (ISI) of $<80 \mathrm{~ms}$ indicating the initiation of a burst, and two spikes with an ISI $>160$ ms signaling burst termination (Grace and Bunney, 1984). To test the effect of noxious stimuli, repeated footshocks were administered (see above) and the activity of the DA neuron was recorded continuously both during the footshocks as well as during the intershock interval. At the cessation of the footshock protocol, recording continued to assess the effect of repeated footshock on VTA DA neuron population activity. Population activity was also assessed in a separate group of rats that had received restraint stress (see above). The effect of these stressors on VTA DA neuron population activity was assessed by passing the electrode through the VTA in a predetermined grid pattern of six to nine tracks separated by $0.2 \mathrm{~mm}$; all spontaneously active DA neurons encountered per electrode track were counted and their firing rate and percentage burst firing determined. In one set of rats, subsequent tracks were made from the medial to the lateral VTA (ML group), while in the second set of rats, recordings were made in a similar pattern but beginning with the lateral VTA and progressing medially (LM group). The three tracks aligned along the measurement of $0.6 \mathrm{~mm}$ from bregma were defined as "medial" VTA; similarly, the three tracks recorded at $1 \mathrm{~mm}$ from bregma were defined as "lateral."

At the end of the experiments, three parameters of VTA DA neuron activity were calculated for each rat; first, the "population activity," defined as the number of spontaneously active VTA DA neurons encountered in each of the completed tracks (i.e., total number of neurons/ number of tracks). In addition, all firing rate and percentage of burst firing of all of the DA neurons recorded in each rat were averaged to obtain the average firing rate (FR) and average percentage burst firing (\%B firing) for the DA neurons for a given animal, respectively. The three parameters obtained from one rat were then averaged among up to 18 rats per condition.

Immunohistochemistry and pharmacological manipulation of the vHPC. The activity of brain regions known to affect DA neuron activity states and their modulation by stressors was examined using immunocytochemical localization of $c$-fos protein. Immunohistochemical studies were performed as described previously (Knapska and Maren, 2009) using commercially available antibodies. Following either $1 \mathrm{~h}$ of acute restraint stress (see above) or no stress, adult male rats were overdosed with sodium pentobarbital ( $120 \mathrm{mg} / \mathrm{kg}$, i.p.) and perfused transcardially with saline $(50 \mathrm{ml})$ followed by paraformaldehyde $(150 \mathrm{ml}, 4 \% \mathrm{w} / \mathrm{v}$ in $0.1 \mathrm{M}$ PBS). Rats were decapitated and their brains removed, postfixed for $24 \mathrm{~h}$ ( $4 \% \mathrm{w} / \mathrm{v}$ paraformaldehyde in $0.1 \mathrm{M}$ PBS), and stored in $0.1 \mathrm{M}$ PBS until sectioning. Free-floating coronal sections $(60 \mu \mathrm{m})$ containing the prefrontal cortex (PFC) or HPC were cut using a freezing microtome. Slices were preblocked with $2 \% \mathrm{v} / \mathrm{v}$ normal goat serum (NGS) in PBS containing $0.3 \% \mathrm{v} / \mathrm{v}$ Triton $\mathrm{X}-100$ for $30 \mathrm{~min}$ at room temperature followed by $3 \times 10 \mathrm{~min}$ washes in PBS. Sections were incubated at $4^{\circ} \mathrm{C}$ for $48-72 \mathrm{~h}$ in PBS containing a rabbit anti-c-fos antibody (PC38; Calbiochem) at a concentration of 1:3000. Sections were washed $(3 \times 10 \mathrm{~min})$ and incubated for $1 \mathrm{~h}$ with biotinylated horse anti-rabbit-IgG $1 / 250$ (containing $1 \%$ NGS $0.3 \%$ Triton X-100) at $37^{\circ} \mathrm{C}$. Color was developed with nickel-enhanced 3,3'-diaminobenzidine following the Vectastain ABC method (Vector Laboratories).

The role of vHPC in modulating the response to stress was tested by local inactivation with TTX followed by single-unit recordings. Acute surgery was performed by drilling another hole overlying the vHPC (AP, -6; ML, +5.3; DV, -5.5), and a stainless steel guide cannula (26 ga; Plastics One Inc.) was implanted to allow local infusion of TTX ( $1 \mu \mathrm{M}, 0.5$ $\mu \mathrm{l})$ or Dulbecco's PBS (dPBS) $(0.5 \mu \mathrm{l})$. All infusions occurred through a microinfusion injector (Plastics One) extending $2 \mathrm{~mm}$ ventral from the guide cannula, and connected to a $5 \mu$ l glass syringe (Hamilton Com- 
pany, model 88011). The speed of TTX administration was controlled with a BAS syringe pump (Bioanalytical Systems Inc, model MD1001) to allow slow infusions over a minute time period. Given that infusion of dPBS did not affect population activity in either control group, the data from the untreated control and the APBS groups (for both control and stress rats) were combined.

Survival surgery and locomotor response to psychostimulants. Survival surgery procedures were conducted in a semi-sterile environment as described previously (Lodge and Grace, 2007) with some modifications. Animals were anesthetized by injection of sodium pentobarbital (Nembutal; $50 \mathrm{mg} / \mathrm{kg}$, i.p.) and placed in a stereotaxic apparatus equipped with blunt atraumatic ear bars. Guide cannulae (22 ga; Plastics One Inc.; AP, -6; ML, +5.3; DV, -5.5) with a cylindrical plastic pedestal molded around a $5 \mathrm{~mm}$ stainless steel tubing were implanted bilaterally in the vHPC and affixed in place with dental cement and four anchor screws (Fine Science Tools). Once the cement solidified, the wound was sutured and the rats were removed from the stereotaxic frame and placed on a thermal heating pad in a clean cage. Rats were administered antibiotics (3 mg/kg gentamicin, s.c.) and postoperative analgesia $(1 \mathrm{mg} / \mathrm{kg}$ ketoprofen, i.m., and 5\% $\mathrm{v} / \mathrm{w}$ Children's Tylenol syrup added to rat chow). Following recovery from anesthesia, all rats were transferred to a reverse light/dark cycle facility (lights on 7:00 P.M.-7:00 A.M.) where they were housed for $\sim 15 \mathrm{~d}$ before behavioral testing, with tests performed during the active part of the diurnal cycle. On the day of the experiment, clusters of four rats segregated into groups of two were randomly assigned to the control or AR group, in which the two AR rats received $2 \mathrm{~h}$ restraint stress in a room with dark or red light on. To maintain consistency with the timing of the electrophysio-

logical recordings, both AR and control rats were housed in the same room for another $2 \mathrm{~h}$. This post-restraint interval was found to yield the most reliable and consistent results with respect to measuring the effect of restraint stress on the activity of DA neurons. Rats were then transported to a different room for behavioral testing; TTX $(1 \mu \mathrm{M}, 0.5 \mu \mathrm{l})$ was infused bilaterally in the vHPCs of one rat in each group (one AR, one control) whereas the other two rats were infused with $0.5 \mu \mathrm{l}$ of dPBS. Following infusion, the four rats were placed in four separate open-field arenas (Coulbourn Instruments), and locomotor activity in the $x-y$ plane was assessed by measuring beam breaks (TruScan Software; Coulbourn Instruments). First, 30 min of spontaneous locomotion was recorded; all four rats were then injected intraperitoneally with $1.5 \mathrm{mg} / \mathrm{kg} \mathrm{D}$-amphetamine, and drug-induced locomotor behavior was recorded for the following $120 \mathrm{~min}$.

Histology. At the cessation of each experimental procedure, all rats were deeply anesthetized with an overdose of chloral hydrate or Nembutal. For electrophysiological recordings, recording sites were marked via electrophoretic ejection of Pontamine sky blue dye from the tip of the recording electrodes ( $\sim-20 \mu \mathrm{A}$ constant current, for 20-30 min; bipolar constant current source; Fintronics Inc.).

Rat brains were removed and placed in $8 \% \mathrm{w} / \mathrm{v}$ paraformaldehyde in PBS for a minimum of $48 \mathrm{~h}$; brains were then cryoprotected with $25 \%$ $\mathrm{w} / \mathrm{v}$ sucrose in PBS until saturated and were then sectioned into $60 \mu \mathrm{m}$ coronal sections with reference to a stereotaxic atlas (Paxinos and Watson, 1998). Brain slices were then mounted onto gelatin chrome-alumcoated slides, and stained with cresyl violet for histochemical verification of electrode and/or cannula sites.
(2)

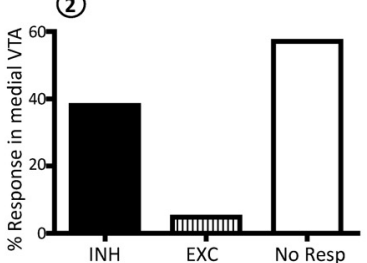

(2)

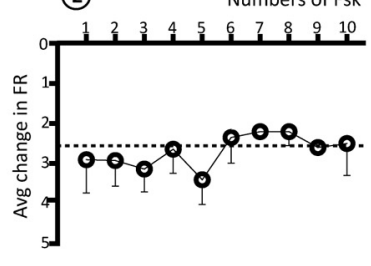

(2)

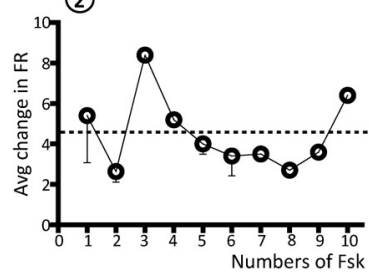

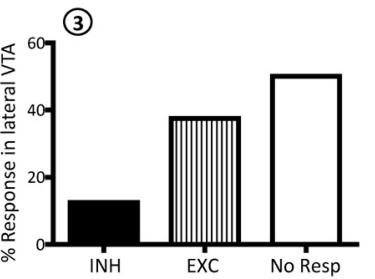

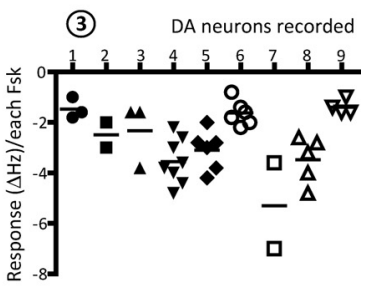

(3)

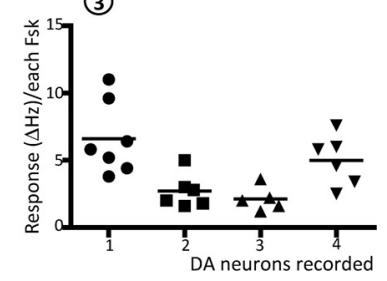

Figure 1. Repeated noxious stimuli induced both inhibition and excitation of VTA DA neurons. $\boldsymbol{A}$, Effects of footshock on identified DA neuron activity. A1, Approximately $45 \%$ of DA neurons tested responded to footshock, and displayed either inhibition ( across each footshock; dashed horizontal line represents average mean change in FR. B3, Individual neurons showed ndividual neurons showed marked differences in amplitude of response, within a given neuron the responses were more tightly clustered in amplitude. $\boldsymbol{C}$, Same as $\boldsymbol{B}$, but for neurons that were excited by footshock.

Data analysis and statistics. The electrophysiological activity of VTA DA neurons was analyzed off-line using Neuroscope. All statistics were performed on raw data using SigmaStat 3.1 (Systat Software Inc.). In electrophysiology experiments, the response to footshock was evaluated in terms of changes in FR, and a change of 2 SDs was used as the criterion for distinguishing responsive from nonresponsive DA neurons. To study changes in population activity, rats were defined as "DA activated" if the value of VTA DA neuron population activity was significantly greater [i.e., lay above the $95 \%$ confidence interval (CI)] compared with mean population activity recorded in control rats; the range of CI values obtained from controls was $0.9-1.2$ cells/track. The effects of acute stress were analyzed using one-way ANOVA or Kruskal-Wallis one-way ANOVA on ranks; the effects of repeated stress were analyzed using one-way ANOVA with repeated measures (RM). A two-way ANOVA was used to analyze multiple comparisons in behavioral studies, with manipulation (controls vs stress) as the between-subjects factor and treatment as the within-subjects factor.

All data are reported as mean \pm SEM.

\section{Results}

Footshock evokes both inhibitory and excitatory responses in VTA DA neurons

Microdialysis studies show that repeated footshock induces a prominent and time-locked DA increase in postsynaptic targets (Saulskaya and Marsden, 1995). By using a similar protocol, the response of VTA DA neurons to single and repeated noxious 

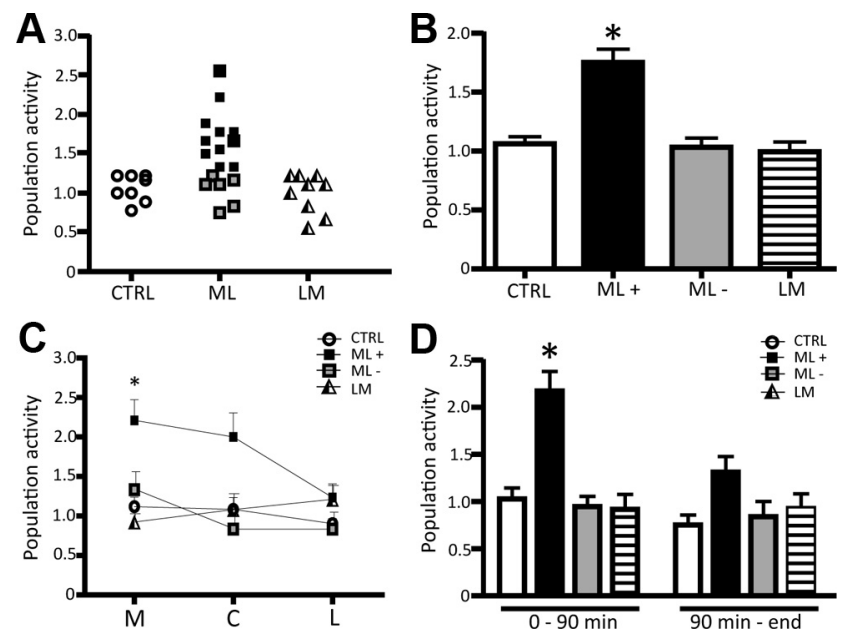

Figure 2. Repeated footshock (Fsk) stimulation causes a selective increase in population activity of medial VTA DA neurons. $\boldsymbol{A}$, Distribution of DA neuron population activity (number of spontaneously active DA neurons encountered per electrode track) for control (CTRL) rats (open circles), and group ML (black squares, DA activated; gray circles, DA nonactivated) and LM (triangles) of rats receiving footshock (each symbol represents the response in a single rat). $\boldsymbol{B}$, The ML group of rats was segregated into two groups: those rats showing a significant increase in population activity ( $\mathrm{ML}+$; black bar) and those that were DA nonactivated (ML - ; gray bar). Recordings made in LM (horizontal line bar) rats did not reveal differences in population activity compared to control (white bar). C, When the regions are analyzed separately, a significant increase in population activity is observed in the medial $(M)$, but not in the central $(C)$ or lateral (L) VTA. $\boldsymbol{D}$, The increase in population activity was also limited in duration, in that significant differences were only observed within 90 min following delivery of the footshock. Significant difference between Fsk rats and CTRL (one-way RM ANOVA); for all values, ${ }^{*} p<0.001$.

stimuli was examined in anesthetized rats. A single footshock evoked a response in $44.8 \%$ of the neurons examined (13 of 29), with $31 \%$ of the neurons inhibited and $13.8 \%$ excited (Fig. 1 A1). Neurons located in the medial VTA exhibited predominantly inhibition during footshock, in that $38 \%$ of the neurons tested $(8$ of 21) were inhibited and only $4.8 \%$ excited (1 of 21) (Fig. 1A2). In contrast, neurons located in the lateral VTA exhibited an opposite response, with $37.5 \%$ ( 3 of 8 ) of neurons excited while only $12.5 \%$ ( 1 of 8 ) were inhibited (Fig. 1 A3). There was no significant difference in the baseline proprieties of the responsive versus nonresponsive DA neurons, both in terms of firing rate (one-way ANOVA, $p=0.425$ ), percentage burst firing (Kruskal-Wallis one-way ANOVA, $p=0.431$ ) or spike train ISI (one-way ANOVA, $p=0.364$ ). Footshock-evoked inhibition displayed a rapid onset and an average duration of $1.2 \pm 0.1 \mathrm{~s}$ (Fig. 1 B1). The evoked inhibition in firing rate averaged $85.1 \pm 4.0 \%$ from baseline activity (\% change range: $62.9-100 \%$ ) with a change in firing rate $(\Delta \mathrm{FR})$ of $-2.7 \pm 0.1 \mathrm{~Hz}$ (Fig. $1 \mathrm{~B} 2$ ). During repetitive presentation of each footshock, none of the neurons responded to all 10 footshocks; inhibited neurons responded to footshocks $49.5 \pm$ $6.1 \%$ of the time (range: $28.6 \%$ to $80 \% ; n=9$ DA neurons). In addition, three of nine neurons responded to the first three footshocks only and then showed accommodation (Fig. 1B3). However, when the DA neuron responded, the response was consistent in both direction and magnitude (one-way RM ANOVA, $p=0.995)$. On the other hand, when comparing across neurons the responses to footshock stimulation were quite variable, with individual neurons exhibiting consistent inhibition that varied significantly when compared between neurons (oneway RM ANOVA, $F_{(7,23)}=7.761, p<0.001$; Fig. 1 B3).

The footshock-evoked excitation had a rapid onset but a longer duration compared with the inhibition, lasting $1.7 \pm 0.2 \mathrm{~s}$
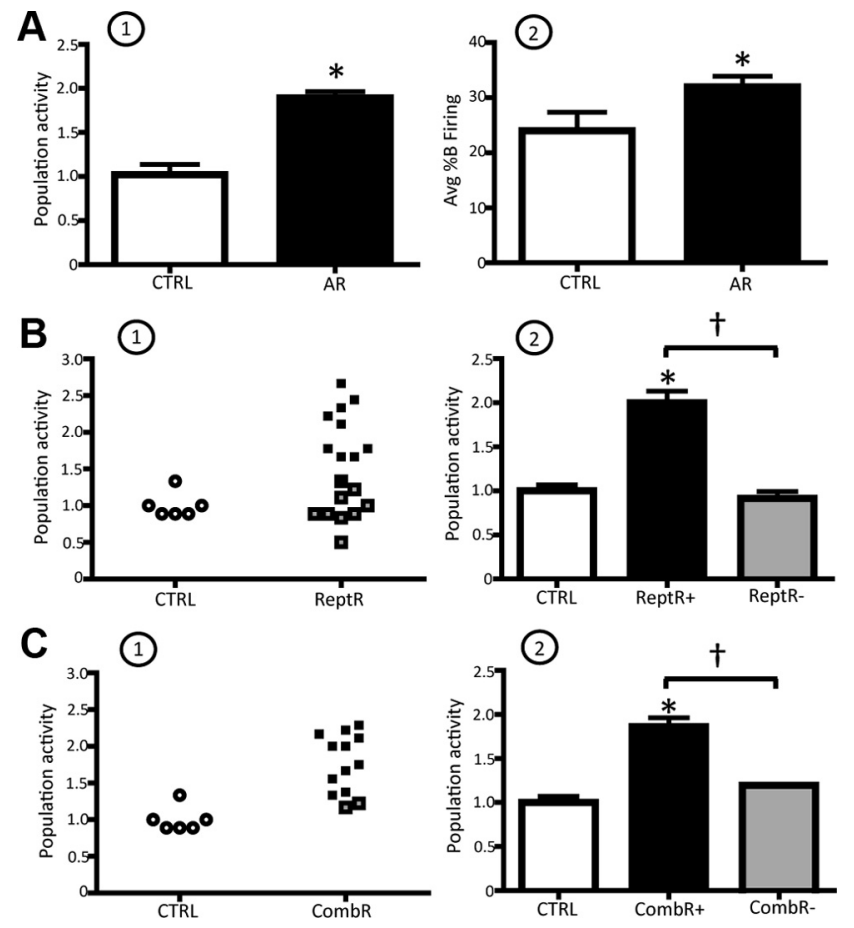

Figure 3. Restraint stress potently activates VTA DA neurons. A1, A2, AR induced a pronounced increase in the average population activity $(\boldsymbol{A} \mathbf{1})$ and in the average percentage burst firing (A2) of the VTA DA neurons recorded. B1, Distribution of population activity observed in control (CTRL; open circles) versus ReptR; DA-activated rat data are shown in black; DAnonactivated data are shown in gray (each symbol represents the response in a single rat). $\mathbf{B}$, ReptR induced a twofold increase in population activity in DA-activated rats, but not in the non-DA-activated group. C, Effect of CombR (repeated plus acute) on VTA DA neuron population activity. Color code: white, controls; black, DA activated following restraint; gray, DA nonactivated. *Significant difference between CTRL and restraint rats; ${ }^{\dagger}$ significant difference in repeated restraint in DA-activated vs -nonactivated rats. Population activity, CTRL vs AR: one-way ANOVA, $p<0.001$; CTRL vs ReptR: one-way RM ANOVA, $p<0.001$; CTRL vs CombR: one-way RM ANOVA, $p<0.001$. Avg \%B firing, CTRL vs AR: one-way ANOVA, $p=0.043$.

( $n=4$ DA neurons; Fig. 1C1). Neurons excited by footshock displayed a uniform magnitude of response during each footshocks, with $110.1 \pm 12 \%$ increase in firing rate (\%change range: 63.2-191\%; one-way RM ANOVA, $\left.F_{(3,11)}=1.137, p=0.413\right)$ and a $\Delta \mathrm{FR}$ of $4.5 \pm 0.6 \mathrm{~Hz}$ (Fig. 1C2). Excited neurons responded to footshock $63.7 \pm 5.5 \%$ of the time, but none of the neurons tested showed accommodation. Moreover, as observed with inhibited neurons, footshock induced different amplitudes of excitation in different neurons (one-way RM ANOVA, $F_{(6,14)}=6.799, p<0.05$; Fig. 1C3).

Repeated footshock stimulation selectively activates medial VTA DA neurons in a time-limited manner

After testing footshock stimulation on single neurons, the number of spontaneously active DA neurons encountered in each track (population activity) was assessed by using two patterns of tracks (Fig. 2). Rats were randomly divided into two groups: one group was recorded beginning with medial tracks first (ML) and the second group was recorded beginning with lateral tracks (LM; see Materials and Methods). No difference in population activity was observed between the two groups in control rats; thus, data were combined. Population activity averaged $1.1 \pm 0.1 \mathrm{DA}$ neurons/track, with the neurons exhibiting an average firing rate of $3.7 \pm 0.2 \mathrm{~Hz}$ and average percentage of burst firing of $21.1 \pm$ $2.5 \%$ ( $n=8$ rats, $n=68$ DA neurons), which was consistent with previous studies (Lodge and Grace, 2007; Valenti and Grace, 2010). In contrast, rats that received footshock were discrimi- 
nated into DA activated and DA nonactivated on the basis of a CI value (see Materials and Methods). Repeated footshock induced a significant increase in population activity in 11 of the 17 rats of the ML group (ML: $1.8 \pm 0.1, n=11$ rats, $n=169$ DA neurons; one-way RM ANOVA $F_{(10,20)}=15.125, p<0.001$; Fig. $2 A, B)$, while the remaining six ML rats exhibited no change in population activity (one-way RM ANOVA, $p=0.789$; Fig. $2 B)$. However, footshock did not affect population activity in any recordings where the VTA was sampled starting with the lateral neurons (one-way RM ANOVA, $p=0.765$; Fig. $2 A, B$ ). When the effects of footshocks were compared on the three medial tracks versus the three central tracks and the three lateral tracks (Fig. 2C), in all DA-activated ML rats repeated footshock increased the population activity in the three medial tracks to more than double the value of controls (CTRL: $1.0 \pm 0.1, n=7$ rats; Footshock (Fsk): $2.2 \pm 0.2, n=11$ rats; one-way RM ANOVA, $\left.F_{(10,19)}=12.712, p<0.001\right)$, without significantly affecting population activity of DA neurons in the three central tracks (one-way RM ANOVA, $p=0.071$ ), or the lateral VTA (one-way RM ANOVA, $p=0.799$; Fig. 2C). Moreover, footshock stimulation increased population activity in a time-limited manner, with a significant effect induced within the first $90 \mathrm{~min}$ following delivery of the footshocks $\left(\mathrm{CTRL}_{0-90^{\prime}}: 1.0 \pm 0.1, n=6\right.$ rats, Fsk $_{0-90^{\prime}}: 2.2 \pm 0.2, n=11$ rats; one-way RM ANOVA, $F_{(10,18)}=13.477, p<0.001$; Fig. $\left.2 D\right)$, which then decayed to control levels (one-way RM ANOVA, $p=0.311$; Fig. $2 D$ ). However, footshock did not affect the average firing rate (one-way RM ANOVA, $p=0.346$ ) or the percentage burst firing (one-way RM ANOVA, $p=0.103$ ) in any of the groups examined. Given that in the LM rats the medial tracks were recorded later than the 90 min time point, it is not surprising that no significant change in the medial tracks was found in this group.

Together, our data indicate that single DA neurons respond consistently to repeated footshock in a manner similar to single footshock, and are mostly inhibited. In contrast, repeated footshock increased DA neuron population activity and, consistent with microdialysis reports (Abercrombie et al., 1989; Kalivas and Duffy, 1995), this effect was more prominent in medially located DA neurons (that project to ventromedial striatal regions) (Ikemoto, 2007) and occurred only within the first $90 \mathrm{~min}$ following stimulation (Saulskaya and Marsden, 1995).

\section{Acute restraint stress potently activates VTA DA neuron population}

In addition to the noxious stimulus, the effects of a psychological stressor on the activity of VTA DA neurons were examined by testing the response to restraint administered both acutely and repetitively.

At the termination of $\mathrm{AR}$, a robust and reliable increase in VTA DA neuron population activity was observed (CTRL: $1.0 \pm$ $0.1, n=5$ rats, $n=41$ DA neurons; AR: $1.9 \pm 0.1, n=11$ rats, $n=$ 180 DA neurons; one-way ANOVA, $F_{(1,14)}=44.843, p<0.001$;
Fig. 3A1); thus, all rats were classified as DA activated. In contrast to footshock, the restraint-induced increase in population activity was uniform independent of the relative location within the VTA (medial: $1.8 \pm 0.2$, central: $1.8 \pm 0.2$, lateral: $1.8 \pm 0.3 ; n=$ 11 rats, one-way ANOVA, $p=0.995)$. In addition, the effect on DA neuron population activity lasted longer than the effects induced by footshock; i.e., for the entire duration of the experiment $(\sim 4-5 \mathrm{~h})$. Acute restraint did not affect the average firing rate (one-way ANOVA, $p=0.337$ ); however, a significant increase in the average percentage burst firing was observed (CTRL: $24 \pm 3.4 \%$, AR: $31.9 \pm 1.9 \%$; one-way ANOVA $F_{(1,14)}=4.963, p=0.043$; Fig. 3A2).

\section{Repeated and combined restraint stress significantly increase VTA DA neuron population activity and burst firing}

The effects of repeated restraint on VTA DA neuron activity were examined $24 \mathrm{~h}$ after the final day of 10 consecutive days of $1 \mathrm{~h}$ restraint. Similar to repeated footshock, at the cessation of the stress paradigm rats were characterized as DA activated $($ ReptR +$)$ and DA nonactivated (ReptR-; Fig. 3B1,2). Repeated restraint stress induced a significant increase in DA neuron population activity in only $56 \%$ (10 of 18 ) of the rats tested (CTRL: $1.0 \pm 0.1, n=6$ rats, $n=54$ DA neurons; ReptR $+: 2.0 \pm 0.1, n=$ 10 rats, $n=176$ DA neurons; ReptR-: $0.9 \pm 0.1, n=63 \mathrm{DA}$ neurons; one-way RM ANOVA, $F_{(9,12)}=49.621, p<0.001$; Fig. $3 B 1,2)$. Similar to that observed with acute restraint, the repeated restraint-induced increase in population activity occurred independent of the DA neuron location within the VTA and was stable throughout the experimental recording time (medial: $2.2 \pm 0.2$, central: $2.1 \pm 0.3$, lateral: $1.7 \pm 0.3 ; n=10$ rats; 
A

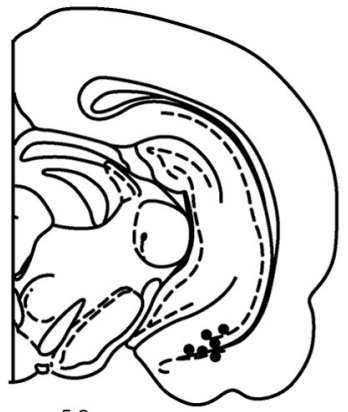

$-5.8 \mathrm{~mm}$

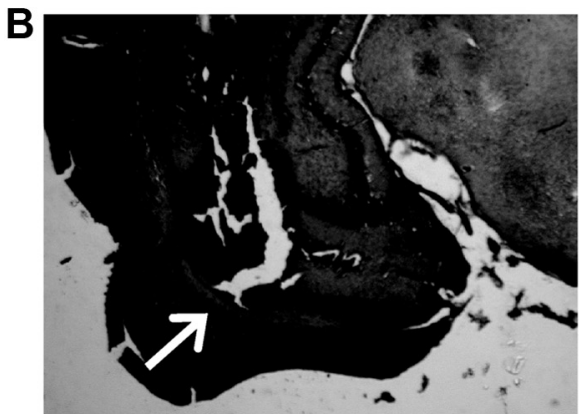

$-6 \mathrm{~mm}$
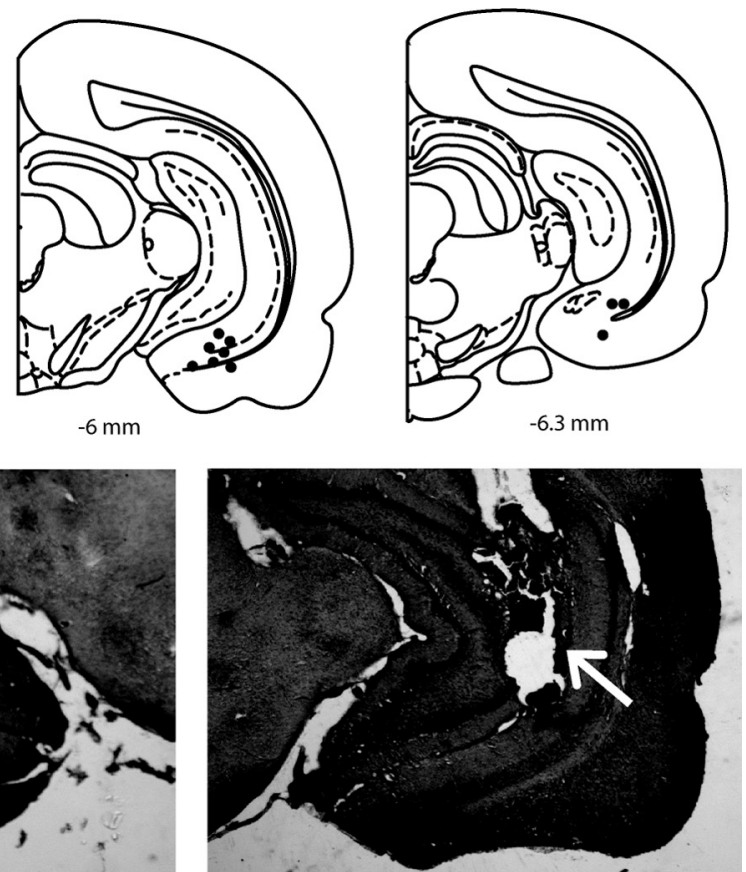

Figure 5. Location of cannulae placements for vHPC inactivation in electrophysiology and behavioral experiments. $\boldsymbol{A}$, Representative localization of cannulae placements (filled dots) in rat vHPC coronal sections for local infusion of $1 \mu \mathrm{M}$ TTX (0.5 $\mu$ ) (electrophysiology experiments). $\boldsymbol{B}$, Arrows indicate location of bilateral cannula placements for TTX infusion in representative behavioral experiment in the left and right $\mathrm{VHPC}$, respectively.

one-way RM ANOVA, $p=0.382)$. Moreover, no change was observed in either the average firing rate (one-way RM ANOVA, $p=0.644$ ) or the average percentage burst firing (one-way RM ANOVA, $p=0.142$ ) across all rats exposed to repeated restraint.

Given that both repeated and acute restraint altered DA neuron population activity, we examined whether the exposure to repeated restraint altered the DA neuron population response to subsequent acute restraint. Thus, a subset of rats was exposed to the combined restraint stress procedure (i.e., repeated followed by acute restraint; see Materials and Methods). According to the defined criteria, 11 of 13 rats tested were DA activated, and the magnitude of increase in population activity was comparable to the two other restraint paradigms (CTRL: $1 \pm 0.1, n=6$ rats, $n=54$ DA neurons; CombR $+: 1.9 \pm 0.1, n=11$ rats, $n=160$ DA neurons; CombR-: $1.2, n=2$ rats, $n=18$ DA neurons; one-way RM ANOVA, $\mathrm{F}_{(10,6)}=40.520, p<0.001$; Fig. 3C1,2). In addition, combined restraint increased population activity uniformly across the medial-lateral extent of the VTA (medial: $1.9 \pm 0.2$, central: $1.9 \pm$ 0.2 , lateral: $1.8 \pm 0.3 ; n=11$ rats; one-way RM ANOVA, $p=0.865$ ) and did not alter the average firing rate (one-way RM ANOVA, $p=0.713$ ) or the average percentage burst firing (one-way RM ANOVA, $p=0.290$ ).

Acute restraint induced a significant increase in c-fos activation in the vHPC

Together, these data suggest that both noxious and psychological stressors induce a pronounced activation of VTA DA neurons manifested as an increase in VTA DA neuron population activity and, with respect to restraint, an increase in burst firing. Our previous work showed that population activity is selectively regulated by the vHPC (Floresco et al., 2001). Therefore, we tested whether the stress-induced increase in population activity was associated with hyperactivity within the vHPC. Exposure to $1 \mathrm{~h}$ restraint stress induced a prominent increase in $c$-fos expression

in the $\mathrm{vHPC}$ (control $=5$ rats, restraint $=$ 5 rats; Fig. $4 A$ ) but did not alter $c$-fos levels in the dorsal HPC (Fig. $4 B$ ). In addition, an increase in $c$-fos expression was observed in the medial PFC (Fig. 4C), a region known to be involved in modulating stress responsivity (Rosenkranz et al., 2003) and that receives potent input from the vHPC (Jay and Witter, 1991).

\section{Local infusion of TTX into the vHPC} reversed the effects of noxious stimuli on VTA DA neuron activity

Given the histochemical evidence for vHPC activation, we examined whether the vHPC was necessary for the stressinduced increase in DA neuron population activity. The $\mathrm{vHPC}$ was inactivated by local infusion of TTX (Fig. 5A) (Lodge and Grace, 2008) both prior or following delivery of footshocks, and the response of VTA DA neurons examined. Infusion of TTX did not alter in any way the effect of footshocks on identified DA neurons (data not shown).

When infused into the vHPC of sham rats, TTX did not affect the number of spontaneously active DA neurons (TTX: $0.9 \pm 0.1 ; n=4$ rats, $n=31$ DA neurons; one-way ANOVA, $p=0.322$ ), average firing rate (TTX: $3.1 \pm 0.7$ $\mathrm{Hz}$; Kruskal-Wallis one-way ANOVA on Ranks, $p=0.461$ ) or average percentage burst firing (TTX: $29.4 \pm 2.5 \%$; one-way ANOVA, $p=0.067$ ) compared with controls, which is consistent with previous observations (Lodge and Grace, 2007). In contrast, following 10 repeated footshock stimulations, local administration of TTX into the vHPC both prevented (when administered before footshocks, $n=6$ rats) or reversed (when administered following footshocks, $n=3$ rats) the footshock-induced increase in population activity of ML rats, thus data were combined (ML: $1.8 \pm 0.1, n=11$ rats, $n=169$ DA neurons; ML + TTX: $1.1 \pm 0.05, n=9$ rats, $n=71$ DA neurons; one-way RM ANOVA, $\mathrm{F}_{(10,8)}=34.696, p<0.001$; Fig. $6 A$ ). Specifically, inactivation of $\mathrm{vHPC}$ prevented the increase in population activity of DA neurons located in the medial VTA of the ML group (ML: $2.2 \pm 0.2, \mathrm{ML}+\mathrm{TTX}: 1.0 \pm 0.1$; one-way RM ANOVA, $\mathrm{F}_{(10,8)}=22.533, p=0.001$; Fig. $\left.6 \mathrm{~B}\right)$ as well as within the first 90 min following footshock (ML: $2.2 \pm 0.2, \mathrm{ML}+$ TTX: $1.0 \pm 0.1$; one-way RM ANOVA, $\mathrm{F}_{(10,8)}=29.186, p<$ 0.001; Fig. 6C).

\section{Inactivation of the vHPC reversed the effects of restraint stress on VTA DA neuron activity}

The effect of vHPC inactivation on the restraint-induced increase in VTA DA neuron population activity was also examined. TTX was infused locally into the vHPC immediately before initiation of single-unit recordings. In rats exposed to acute restraint stress, TTX infusion into the vHPC reversed the stress-induced increase in population activity (AR: $1.9 \pm 0.1, n=11$ rats, $n=180 \mathrm{DA}$ neurons; AR + TTX: $1.0 \pm 0.1, n=8$ rats, $n=71$ DA neurons; one-way ANOVA, $F_{(1,17)}=53.538, p<0.001$; Fig. $\left.7 A\right)$. However, infusion of TTX into the vHPC failed to reverse the acute restraint-induced increase in percentage burst firing (one-way RM ANOVA, $p=0.938$; Fig. $7 B$ ). 

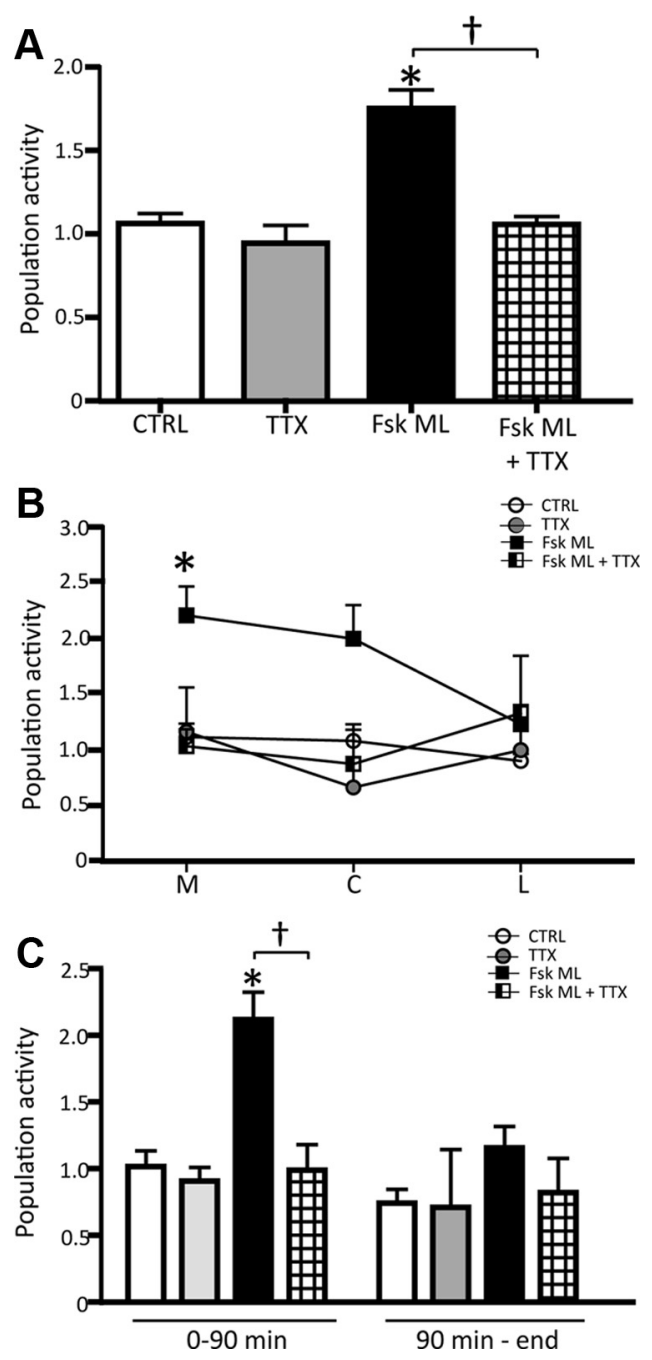

Figure 6. The VHPC is required for the footshock-induced increase in VTA DA neuron population activity. $\boldsymbol{A}-\boldsymbol{C}$, Local infusion of TTX into the VHPC either prevented or reversed (see Results) the footshock-induced increase in DA neuron population activity $(\boldsymbol{A})$ that predominantly occurred in the medial VTA (B) and within the first 90 min following repeated footshocks ( $($ ). Color code for bar diagram and symbols: white, controls; gray, control + TTX; black, footshock; grid, footshock + TTX. Significant difference between footshock (group ML, DA activated only) and CTRL (one-way RM ANOVA), ${ }^{*} p<0.001$; significant difference between footshock (Fsk) ML and Fsk ML + TTX (one-way RM ANOVA), ${ }^{\dagger} p<0.001$.

Similarly, inactivation of the vHPC also reversed the increase in DA neuron population activity occurring following repeated restraint stress (ReptR: $2.0 \pm 0.1, n=10$ rats, $n=176$ DA neurons; ReptR + TTX: $0.9 \pm 0.1, n=9$ rats, $n=63$ DA neurons; one-way RM ANOVA, $F_{(9,8)}=67.424, p<0.001$; Fig. $7 C$ ) and the increased population activity observed following combined restraint (CombR: $1.9 \pm 0.1, n=11$ rats, $n=160$ DA neurons; CombR + TTX: $0.9 \pm$ $0.1, n=8$ rats, $n=57$ DA neurons; one-way RM ANOVA, $\mathrm{F}_{(12,7)}=$ 29.107, $p<0.001$; Fig. 7D). As expected (Lodge and Grace, 2007), infusion of TTX into the vHPC of sham rats did not change any of the DA neuron proprieties compared with matched CTRL (population activity, Kruskal-Wallis one-way ANOVA on Ranks, $p=0.423$; Avg FR, one-way ANOVA, $p=0.549$; Avg \%B Firing, one-way ANOVA, $p=0.082$ ).

Restraint stress potentiates amphetamine-induced locomotor activation in a vHPC-dependent manner

Stress is known to cross-sensitize with amphetamine, in that restraint stress will increase the locomotor response to acute am-
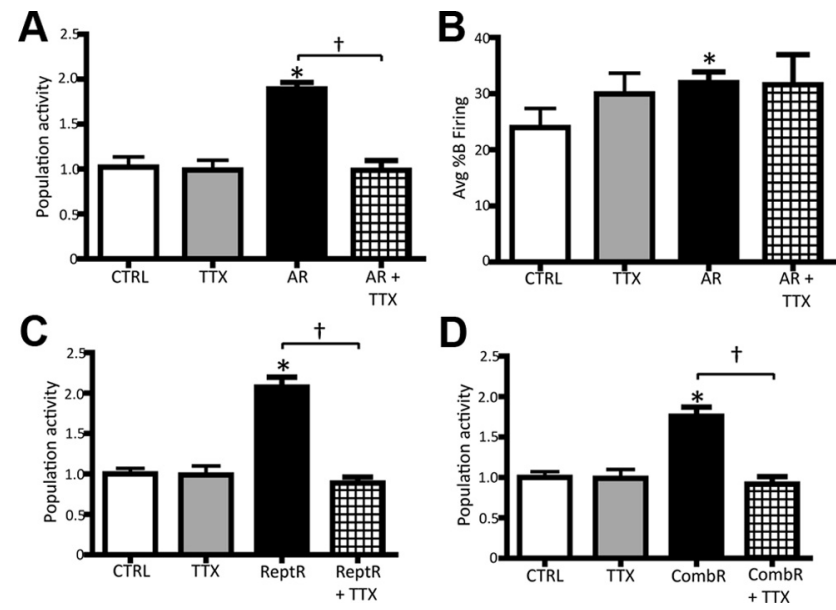

Figure 7. Inactivation of the $\mathrm{VHPC}$ reversed the restraint-induced increase in DA neuron population activity. $\boldsymbol{A}$, Population activity in control (CTRL) and AR rats; TTX administered into the $\mathrm{VHPC}$ following restraint reversed the increase in population activity in the AR rats but did not affect controls. $\boldsymbol{B}$, In contrast, inactivation of the vHPC did not affect the AR-induced increase in percentage burst firing. $C$, The effects of ReptR stress on DA neuron population activity and its reversal by infusion of TTX into the VHPC. $D$, Effect of CombR stress on population activity and its reversal by infusion of TTX. Color code for bar diagram and symbols: white, controls; gray, control + TTX; black, footshock; grid, footshock + TTX. * Significant difference between restraint and CTRL; ${ }^{\dagger}$ significant difference between restraint and restraint + TTX; ARvs AR + TTX: one-way ANOVA, $p<0.001$; ReptRvs ReptR + TTX: one-way RM ANOVA, $p<0.001$; CombR vs CombR + TTX: one-way RM ANOVA, $p=0.001$.

phetamine injection (Antelman et al., 1980; Pacchioni et al., 2007). Furthermore, we have found that the level of DA neuron population activity correlates with the behavioral responses to amphetamine (Lodge and Grace, 2007, 2008). Given that stressors also cause a prominent increase in DA neuron population activity, we examined whether this increase was associated with an increased behavioral response to the drug. In rats that had undergone $2 \mathrm{~h}$ of restraint, the distance traveled in an open field was altered (trend toward less activity) from that observed in control rats; thus, all data are expressed as percentage change from predrug baseline (Fig. 8). Following administration of 1.5 $\mathrm{mg} / \mathrm{kg}$ amphetamine, rats that had been previously exposed to restraint displayed significantly higher levels of locomotion compared with rats infused with dPBS, which corresponded to a $24.3 \%$ higher level of locomotor activity over baseline (CTRL: $1362.1 \pm$ $188.4 \mathrm{~cm}, n=13$ rats; AR: $1799.8 \pm 128.6 \mathrm{~cm}, n=15$ rats; peak: 40 min from amphetamine administration; two-way ANOVA, $\mathrm{F}_{(1,1,836)}=$ 18.984, $p<0.001$; Fig. $8 A$ ). Furthermore, inactivation of the vHPC by bilateral infusion of TTX (Fig. $5 B$ ) resulted in a significant decrease in amphetamine-induced locomotion in restraint rats (AR + TTX: $1618.6 \pm 157.6 \mathrm{~cm}, n=12$ rats; two-way ANOVA, $\mathrm{F}_{(1,1,806)}=8.246, p=0.004$; Fig. $\left.8 \mathrm{~B}\right)$ but not in controls (two-way ANOVA, $p=1.0$; Fig. $8 C$ ). In particular, following inactivation of the vHPC, the amount of locomotion produced by amphetamine was not significantly different in the restraint animals compared with controls. Therefore, following acute restraint, the ability of restraint stress to potentiate amphetamine-induced locomotor behavior was attenuated by inactivation of the vHPC.

\section{Discussion}

The data presented here provide the first physiological explanation regarding how stressful stimuli can increase neurochemical indices of mesolimbic DA activity. We found that, although single footshocks tend to transiently inhibit DA neuron firing, repeated stimuli similar to those that induce increases in DA in the NAc cause 

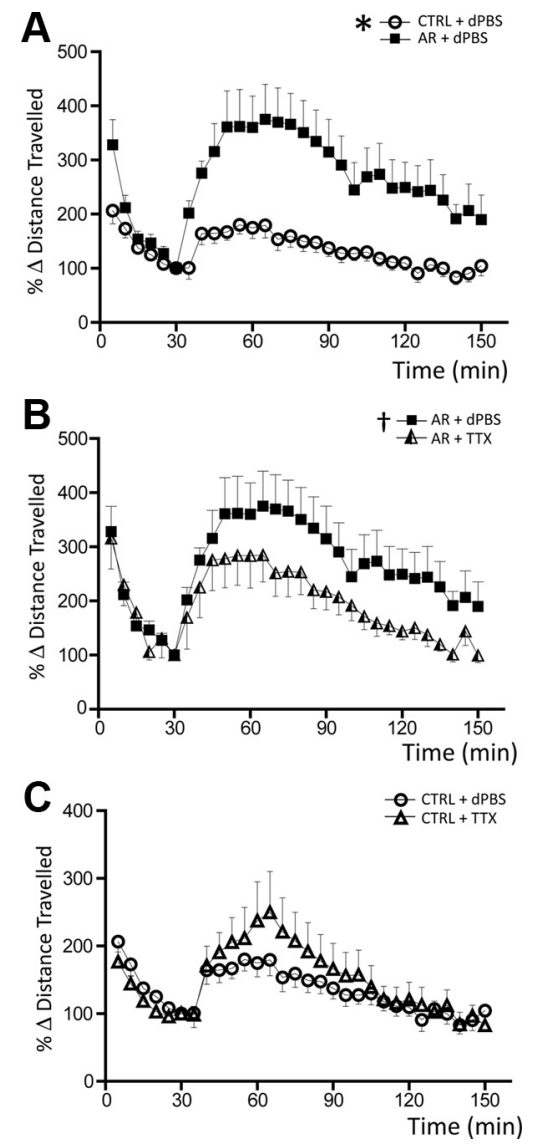

Figure 8. Inactivation of the VHPC reverses the acute restraint-induced potentiation of amphetamine-induced locomotion. $\boldsymbol{A}$, Time course of amphetamine-induced increase in locomotor activity, comparing AR (black squares) and control (CTRL; open circles). In acute restraint animals, administration of $1.5 \mathrm{mg} / \mathrm{kg}$ amphetamine caused a substantially greater increase in locomotor activity compared with nonstressed controls. $\boldsymbol{B}$, Infusion of TTX into the vHPC reversed the stress-induced augmentation of amphetamine-induced locomotion in the AR rats (triangles, AR followed by infusion of TTX). C, Inactivation of the vHPC did not affect the locomotor response to amphetamine in control rats (open triangles; (TRL + TTX). Significant difference between CTRL $+\mathrm{dPBS}$ and AR $+\mathrm{dPBS}$ (two-way ANOVA), ${ }^{*} p<0.001$; significant difference between AR $+\mathrm{dPBS}$ and AR $+\operatorname{TTX}$ (two-way ANOVA), ${ }^{\dagger} p=0.004$.

a pronounced increase in DA neuron population activity in the majority of the rats tested. Moreover, this stress-induced increased in population activity is driven by activation of the vHPC. Thus, restraint causes increased $c$-fos protein in the vHPC, whereas inactivation of the vHPC reversed both the increase in DA neuron population activity and the stress-induced augmentation of amphetamine-induced increase in locomotor activity, supporting a key role of vHPC in modulating stress cross-sensitization with drugs of abuse.

The finding that stressors applied either during anesthesia (i.e., footshock) or in the awake state (i.e., restraint) have similar effects on DA neuron population activity suggest that a conscious state is not required for activation of the vHPC-VTA circuit. Moreover, the change in DA neuron population activity to footshock in the anesthetized rat parallels both the time course and regional specificity of the neurochemical response of the DA system measured in awake animals. However, whether the longterm activation that occurs with restraint is partly due to the fact that this stressor is applied during the conscious state is unclear. We have shown previously that conditioned responses in the amygdala are more robust when the conditioning occurs in the conscious state (Rosenkranz et al., 2003), suggesting that stimuli may have a more robust impact when cognitive processes are intact.
Although the DA system has long been known to play a role in reward and novelty, its function in responding to aversive events has been unclear. Thus, recordings of the activity of single DA neurons to transient noxious stimuli have revealed a predominantly inhibitory response (Bunney et al., 1973; Grace and Bunney, 1979; Schultz and Romo, 1987; Brischoux et al., 2009), and excitation in a few cases (Ungless et al., 2004). This increase in DA neuron population activity is consistent with microdialysis studies that demonstrated a significant increase in DA levels in postsynaptic targets of DA neurons following presentation of aversive stimuli. Moreover, the stimulus-induced increase in DA neuron population activity produced time-limited effects that were also regionally selective, in that only medial VTA DA neurons showed increased population activity to footshock. This result correlates with microdialysis reports that indicate a specific increase in DA levels in NAc shell, but not in the core (Abercrombie et al., 1989; Kalivas and Duffy, 1995). Moreover, the stress-induced increase in population activity arose immediately after cessation of footshock and lasted for $\sim 90 \mathrm{~min}$, which is consistent with the timelimited increase in NAc shell DA levels observed in microdialysis studies (Saulskaya and Marsden, 1995).

In contrast to responses to noxious stimuli, more complex psychological stressors such as restraint induce prolonged changes in DA system responsivity. Thus, studies show that $2 \mathrm{~h}$ of restraint stress increase the behavioral response to amphetamine for $24 \mathrm{~h}$ following the exposure (Pacchioni et al., 2007). Therefore, we examined how $2 \mathrm{~h}$ of restraint affected DA neuron population activity. In contrast to footshock, the restraint stress-induced increase in DA neuron population activity was both sustained and was uniform across the medial-lateral extent of the VTA. Interestingly, while acute restraint consistently increased DA neuron population activity across all animals tested, if the restraint was repeated across $10 \mathrm{~d}$ the rats, as with repeated footshock, fell into two groups: a DA-activated and a DA-nonactivated group. The reason for this difference in response is not clear, but may be due to individual differences in the ability of animals to accommodate to repeated stressful events (Bhatnagar et al., 2004). Another difference with restraint stress is the increase in burst firing observed with acute stress. This is consistent with previous reports showing that restraint stress in awake behaving animals also led to increased burst firing in DA neurons (Anstrom and Woodward, 2005). While the origin of burst firing following restraint is not clear, it appears to be mediated via a different pathway than that mediating the increase in population activity, since inactivation of the vHPC reversed restraint stress-induced increases in DA neuron population activity without affecting burst firing. This is also consistent with our studies showing that population activity and burst firing are mediated by distinct afferent systems (Floresco et al., 2003; Lodge and Grace, 2006b).

Previous studies from our laboratory showed that DA neuron population activity is regulated by the vHPC, via a vHPC-NAcventral pallidum-VTA pathway (Floresco et al., 2001). Modulation of DA neuron population activity has been proposed to determine the "gain" of the DA system (Lodge and Grace, 2006b), in that phasic DA neuron burst firing will only occur in DA neurons that are already firing spontaneously (Floresco et al., 2001). Thus, an increase in the number of DA neurons firing will increase the amplitude of response to phasic events (Lodge and Grace, 2006a). This model is also consistent with the role of the vHPC in context-dependency, in that the responsivity of the DA system should be functionally related to the demands of the context (Grace et al., 2007). Stress is a context-dependent process, in that the response of an animal to an acute stressor is dependent 
on the context (Bouton and Bolles, 1979; Bouton and King, 1983). Indeed, we have shown that amphetamine sensitization, which is also context-dependent (Vezina et al., 1989; Crombag et al., 2000), is also attenuated by inactivation of the vHPC (Lodge and Grace, 2007). Similarly, we showed here that stress-induced increases in DA neuron population activity depend on the vHPC. Thus, restraint stress increases $c$-fos protein in the vHPC, and inactivation of the vHPC prevents or reverses the increase in DA neuron population activity in response to repeated footshock and restraint stress, as well as attenuating the restraint stress-induced augmentation of the behavioral response to amphetamine. This latter finding is consistent with the reported cross-sensitization that occurs with stress and amphetamine (Antelman et al., 1980; Pacchioni et al., 2007).

The dependence of stress-induced amphetamine sensitization on the vHPC is also consistent with studies showing that stress (Shaham and Stewart, 1995) and the vHPC (Vorel et al., 2001) play key roles in mediating relapse to drug-seeking behavior, and the vHPC modulates the amplitude of the behavioral response to psychostimulants (Taepavarapruk and Phillips, 2003; White et al., 2006). Studies show that the vHPC has a central role in orchestrating the response to stress via a regulation of the hypothalamic-pituitary adrenal axis (Herman et al., 1995; Cooper et al., 2006). Stress has also been shown to be a risk factor in the onset and exacerbation of psychosis. Interestingly, in a developmental disruption animal model of schizophrenia, the hyperresponsivity to amphetamine was also found to be dependent on hyperactivity within the vHPC (Lodge and Grace, 2008).

In conclusion, our data are suggestive of a hyperdopaminergic condition associated with stress (either acute or repeated) which we propose is driven by hyperactivity within the vHPC. The mechanism underlying this hyperactivity is not clear, but we have found in preliminary studies that the VHPC is potently excited by stimulation of sites with known involvement in stress, such as the locus ceruleus and the basolateral amygdala (Lipski and Grace, 2008). Indeed, the response of vHPC neurons to footshock was found to correlate with their response to locus ceruleus stimulation (Lipski and Grace, 2009). Therefore, the vHPC appears to be a critical link between context-dependent sensitization of the DA system, stress-induced potentiation of amphetamine responsivity, and the increase in DA levels associated with both repeated noxious or acute psychological stressors. Whether the vHPC is the primary drive of the stress responsivity or more of an enabling system is unclear from the present studies. Nonetheless, the vHPC role in context suggests that this system is not mediating the stress response, but is instead biasing the DA system into a high or low responsive state depending on prior experience within a given context. As such, the VTA would be in a state to show high levels of responsivity to inputs signaling an encounter with a behaviorally salient stimulus (either threatening or rewarding) within this context. The finding that there is an increase in VTA activity for both reward-related events (Lodge and Grace, 2008) and maintained stressors is consistent with a role for VTAaccumbens activation as a means to control vigilance and reactivity depending on the conditions of the environment. Moreover, the finding that stress, drug abuse (Lodge and Grace, 2008), and models of schizophrenia (Lodge and Grace, 2007) all exhibit similar vHPCdependent potentiation of VTA neuron responsivity could provide an explanation for the common role of stress in the exacerbation and relapse in several psychiatric disorders, and therefore may be an effective therapeutic target in the treatment of these disorders.

\section{References}

Abercrombie ED, Keefe KA, DiFrischia DS, Zigmond MJ (1989) Differential effect of stress on in vivo dopamine release in striatum, nucleus accumbens, and medial frontal cortex. J Neurochem 52:1655-1658.

Anstrom KK, Woodward DJ (2005) Restraint increases dopaminergic burst firing in awake rats. Neuropsychopharmacology 30:1832-1840.

Antelman SM, Eichler AJ, Black CA, Kocan D (1980) Interchangeability of stress and amphetamine in sensitization. Science 207:329-331.

Bhatnagar S, Sun LM, Raber J, Maren S, Julius D, Dallman MF (2004) Changes in anxiety-related behaviors and hypothalamic-pituitaryadrenal activity in mice lacking the 5-HT-3A receptor. Physiol Behav 81:545-555.

Bouton ME, Bolles RC (1979) Role of conditioned contextual stimuli in reinstatement of extinguished fear. J Exp Psychol Anim Behav Process 5:368-378

Bouton ME, King DA (1983) Contextual control of the extinction of conditioned fear: tests for the associative value of the context. J Exp Psychol Anim Behav Process 9:248-265.

Brischoux F, Chakraborty S, Brierley DI, Ungless MA (2009) Phasic excitation of dopamine neurons in ventral VTA by noxious stimuli. Proc Natl Acad Sci U S A 106:4894-4899.

Bunney BS, Walters JR, Roth RH, Aghajanian GK (1973) Dopaminergic neurons: effect of antipsychotic drugs and amphetamine on single cell activity. J Pharmacol Exp Ther 185:560-571.

Cooper DC, Klipec WD, Fowler MA, Ozkan ED (2006) A role for the subiculum in the brain motivation/reward circuitry. Behav Brain Res 174:225-231.

Crombag HS, Badiani A, Maren S, Robinson TE (2000) The role of contextual versus discrete drug-associated cues in promoting the induction of psychomotor sensitization to intravenous amphetamine. Behav Brain Res 116:1-22.

Floresco SB, Todd CL, Grace AA (2001) Glutamatergic afferents from the hippocampus to the nucleus accumbens regulate activity of ventral tegmental area dopamine neurons. J Neurosci 21:4915-4922.

Floresco SB, West AR, Ash B, Moore H, Grace AA (2003) Afferent modulation of dopamine neuron firing differentially regulates tonic and phasic dopamine transmission. Nat Neurosci 6:968-973.

Grace AA (2000) Gating of information flow within the limbic system and the pathophysiology of schizophrenia. Brain Res Brain Res Rev 31:330-341.

Grace AA, Bunney BS (1979) Paradoxical GABA excitation of nigral dopaminergic cells: indirect mediation through reticulata inhibitory neurons. Eur J Pharmacol 59:211-218.

Grace AA, Bunney BS (1983) Intracellular and extracellular electrophysiology of nigral dopaminergic neurons-1. Identification and characterization. Neuroscience 10:301-315.

Grace AA, Bunney BS (1984) The control of firing pattern in nigral dopamine neurons: single spike firing. J Neurosci 4:2866-2876.

Grace AA, Floresco SB, Goto Y, Lodge DJ (2007) Regulation of firing of dopaminergic neurons and control of goal-directed behaviors. Trends Neurosci 30:220-227.

Henry DJ, Greene MA, White FJ (1989) Electrophysiological effects of cocaine in the mesoaccumbens dopamine system: repeated administration. J Pharmacol Exp Ther 251:833-839.

Herman JP, Cullinan WE, Morano MI, Akil H, Watson SJ (1995) Contribution of the ventral subiculum to inhibitory regulation of the hypothalamo-pituitary-adrenocortical axis. J Neuroendocrinol $7: 475-482$.

Ikemoto S (2007) Dopamine reward circuitry: two projection systems from the ventral midbrain to the nucleus accumbens-olfactory tubercle complex. Brain Res Rev 56:27-78.

Imperato A, Cabib S, Puglisi-Allegra S (1993) Repeated stressful experiences differently affect the time-dependent responses of the mesolimbic dopamine system to the stressor. Brain Res 601:333-336.

Jarrard LE (1995) What does the hippocampus really do? Behav Brain Res 71:1-10.

Jay TM, Witter MP (1991) Distribution of hippocampal CA1 and subicular efferents in the prefrontal cortex of the rat studied by means of anterograde transport of Phaseolus vulgaris-leucoagglutinin. J Comp Neurol 313:574-586.

Kalivas PW, Duffy P (1995) Selective activation of dopamine transmission in the shell of the nucleus accumbens by stress. Brain Res 675:325-328. 
Knapska E, Maren S (2009) Reciprocal patterns of c-Fos expression in the medial prefrontal cortex and amygdala after extinction and renewal of conditioned fear. Learn Mem 16:486-493.

Lipski WJ, Grace AA (2008) Neurons in the ventral subiculum are activated by noxious stimuli and are modulated by noradrenergic afferents. Soc Neurosci Abstr 34:195.1.

Lipski WJ, Grace AA (2009) Locus coeruleus stimulation and norepinephrine application produce opposite effects on accumbens-projecting neurons in the ventral subiculum. Soc Neurosci Abstr 35:815.1.

Lodge DJ, Grace AA (2006a) The laterodorsal tegmentum is essential for burst firing of ventral tegmental area dopamine neurons. Proc Natl Acad Sci U S A 103:5167-5172.

Lodge DJ, Grace AA (2006b) The hippocampus modulates dopamine neuron responsivity by regulating the intensity of phasic neuron activation. Neuropsychopharmacology 31:1356-1361.

Lodge DJ, Grace AA (2007) Aberrant hippocampal activity underlies the dopamine dysregulation in an animal model of schizophrenia. J Neurosci 27:11424-11430.

Lodge DJ, Grace AA (2008) Amphetamine activation of hippocampal drive of mesolimbic dopamine neurons: a mechanism of behavioral sensitization. J Neurosci 28:7876-7882.

Maren S (1999) Neurotoxic or electrolytic lesions of the ventral subiculum produce deficits in the acquisition and expression of Pavlovian fear conditioning in rats. Behav Neurosci 113:283-290.

Pacchioni AM, Cador M, Bregonzio C, Cancela LM (2007) A glutamatedopamine interaction in the persistent enhanced response to amphetamine in nucleus accumbens core but not shell following a single restraint stress. Neuropsychopharmacology 32:682-692.

Pascucci T, Ventura R, Latagliata EC, Cabib S, Puglisi-Allegra S (2007) The medial prefrontal cortex determines the accumbens dopamine response to stress through the opposing influences of norepinephrine and dopamine. Cereb Cortex 17:2796-2804.

Paxinos G, Watson C (1998) The rat brain in stereotaxic coordinates, Ed 4. New York: Academic.

Piazza PV, Le Moal M (1998) The role of stress in drug self-administration. Trends Pharmacol Sci 19:67-74.

Pierce RC, Kalivas PW (1997) A circuitry model of the expression of behavioral sensitization to amphetamine-like psychostimulants. Brain Res Brain Res Rev 25:192-216.
Rosenkranz JA, Moore H, Grace AA (2003) The prefrontal cortex regulates lateral amygdala neuronal plasticity and responses to previously conditioned stimuli. J Neurosci 23:11054-11064.

Saulskaya N, Marsden CA (1995) Conditioned dopamine release: dependence upon $N$-methyl-D-aspartate receptors. Neuroscience 67: $57-63$.

Schultz W, Romo R (1987) Responses of nigrostriatal dopamine neurons to high-intensity somatosensory stimulation in the anesthetized monkey. J Neurophysiol 57:201-217.

Schultz W, Dayan P, Montague PR (1997) A neural substrate of prediction and reward. Science 275:1593-1599.

Shaham Y, Stewart J (1995) Stress reinstates heroin-seeking in drug-free animals: an effect mimicking heroin, not withdrawal. Psychopharmacology (Berl) 119:334-341.

Sharp PE (1999) Complimentary roles for hippocampal versus subicular/ entorhinal place cells in coding place, context, and events. Hippocampus 9:432-443.

Taepavarapruk P, Phillips AG (2003) Neurochemical correlates of relapse to $d$-amphetamine self-administration by rats induced by stimulation of the ventral subiculum. Psychopharmacology (Berl) 168:99-108.

Thierry AM, Tassin JP, Blanc G, Glowinski J (1976) Selective activation of mesocortical DA system by stress. Nature 263:242-244.

Ungless MA, Magill PJ, Bolam JP (2004) Uniform inhibition of dopamine neurons in the ventral tegmental area by aversive stimuli. Science 303:2040-2042.

Valenti O, Grace AA (2010) Antipsychotic drug-induced increases in ventral tegmental area dopamine neuron population activity via activation of the nucleus accumbens-ventral pallidum pathway. Int J Neuropsychopharmacol 13:845-860.

Vezina P, Giovino AA, Wise RA, Stewart J (1989) Environment-specific cross-sensitization between the locomotor activating effects of morphine and amphetamine. Pharmacol Biochem Behav 32:581-584.

Vorel SR, Liu X, Hayes RJ, Spector JA, Gardner EL (2001) Relapse to cocaine-seeking after hippocampal theta burst stimulation. Science 292:1175-1178.

White IM, Whitaker C, White W (2006) Amphetamine-induced hyperlocomotion in rats: hippocampal modulation of the nucleus accumbens. Hippocampus 16:596-603. 